\title{
Testes de Identidade Generalizada com Objetos em Macaco-Prego (Cebus apella) ${ }^{1}$
}

\author{
Carlos Barbosa Alves de Souza ${ }^{2}$ \\ Rubilene Pinheiro Borges \\ Paulo Roney Kilpp Goulart \\ Romariz da Silva Barros \\ Olavo de Faria Galvão \\ Universidade Federal do Pará
}

\begin{abstract}
RESUMO - Macacos-prego demonstram identidade generalizada (IG) quando os testes são precedidos de treino de discriminações simples com mudanças sucessivas (DSMS) da função dos estímulos usados nos testes. O presente estudo avaliou se esse efeito facilitador deve-se exclusivamente ao contato prévio com os estímulos. Submeteu-se um macaco-prego a quatro testes de IG com objetos: Teste 1 - diretamente com estímulos novos em uma tarefa de discriminação condicional por identidade; Teste 2 - com os mesmos estímulos do teste anterior após o treino de indução de controle condicional via DSMS; Teste 3 - com estímulos novos previamente manipulados livremente pelo sujeito; e Teste 4 - com os mesmos estímulos do Teste 3 após terem sido apresentados em treino de DSMS. O desempenho do sujeito atingiu o critério de aprendizagem nos testes 2 e 4 . O treino de indução de controle condicional via DSMS com os estímulos de teste foi mais efetivo para produzir IG do que a livre manipulação.
\end{abstract}

Palavras-chave: identidade generalizada; discriminação simples; discriminação condicional; macaco-prego.

\section{Generalized Identity Tests with Objects in Capuchin Monkeys (Cebus apella)}

\begin{abstract}
Capuchin monkeys show generalized identity (GI) when tests are preceded by repeated shifts of simple discrimination training (RSSD) with stimuli used in tests. The present study evaluated if that facilitating effect of RSSD training could be due exclusively to the previous contact with the testing stimuli. It was given to a male capuchin monkey four GI tests with objects: Test 1 - an identity conditional discrimination task with new stimuli; Test 2 - with the same stimuli used in Test 1 after RSSD training to induce conditional control; Test 3 - with new stimuli, freely manipulated by the subject previously; and Test 4 - with the same stimuli used in Test 3 after RSSD training. The subject's performance reached the learning criterion in Tests 2 and 4. RSSD training was more effective than free manipulation to produce immediate matching to sample with new stimuli.
\end{abstract}

Keywords: generalized identity; simple discrimination; conditional discrimination; capuchin monkey.

Responder às relações arbitrárias entre estímulos não ensinadas diretamente, de forma que esses estímulos passam a ser substituíveis entre si no controle de repertórios específicos, é uma habilidade que diversas espécies apresentam no processo de adaptação ao seu ambiente (Hauser, Chomsky \& Fitch, 2002; Sidman, 2000). Esse tipo de habilidade é denominado na literatura como comportamento simbólico e tem sido estudado, por exemplo, em primatas humanos e não-humanos (Hauser \& cols., 2002; Hixson, 1998), golfinhos (Herman, Gory, Hovancik \& Bradshaw, 1989) e leões

1 Para a concretização deste trabalho, os autores receberam apoio financeiro do CNPq por meio de Bolsa de Produtividade ao primeiro, quarto e ao quinto autor, Bolsa de Iniciação Científica à segunda autora, e Bolsa de Doutorado ao terceiro autor; da FINEP FNDCT/CT-INFRA, Edital 02/03; e do National Institutes of Health (NIH). Agradecemos a colaboração de Oriana Comesanha na coleta de dados, e o trabalho de manutenção e alimentação dos animais da Escola Experimental de Primatas da UFPA, realizado por Edilson Ferreira Pastana. O software utilizado na coleta de dados foi desenvolvido por Dione Monteiro.

2 Endereço para correspondência: Rod. Arthur Bernardes, 1650. Cond. Alto de Pinheiros, Q.6, Casa 15, Pratinha. Belém, PA. CEP 66825-000. Tel: (91)32489004.E-mail: carlos.souza@pesquisador.cnpq.br. marinhos (Schusterman \& Kastak, 1993). Os estudos mais comuns nessa área com não-humanos, entretanto, têm sido desenvolvidos com primatas, tendo em vista que são mais próximos geneticamente do homem e, portanto, considerados modelos mais apropriados para comparações com a nossa espécie (Tomasello \& Call, 1997).

Savage-Rumbaugh e colaboradores (e.g., Savage-Rumbaugh, 1984; Savage-Rumbaugh \& Lewin, 1994; SavageRumbaugh, McDonald, Sevcik, Hopkins \& Rubert, 1986) têm obtido resultados interessantes ao ensinar repertórios simbólicos para bonobos (Pan paniscus) e chimpanzés (Pan troglodytes) por meio do uso de representações gráficas (lexigramas) de objetos e ações. Em um de seus estudos, Kanzi, um bonobo com 8 anos de idade, apresentou um repertório de compreensão ${ }^{3}$ de sentenças igual ou superior ao de uma criança de 3 anos nas mesmas tarefas (SavageRumbaugh \& cols., 1993).

3 “Compreensão" é aqui entendida como o controle produtivo, ou seja, além do diretamente treinado, pela relação arbitrária entre sentenças e os objetos e eventos a elas relacionados. 
Os resultados de Savage-Rumbaugh e colaboradores fortalecem a possibilidade (e a utilidade) de desenvolver modelos animais da aquisição de repertórios simbólicos que auxiliem na compreensão desses repertórios nos seres-humanos (McIlvane \& Cataldo, 1996). Nesse sentido, além de chimpanzés e bonobos, outra espécie de primata não-humano que vem sendo utilizada em pesquisas sobre comportamento simbólico é o macaco-prego (Cebus apella). Essa espécie faz uso de ferramentas, apresenta respostas discriminadas em relação a ferramentas que podem ser utilizadas e aprende por imitação (Evans \& Westergaard, 2004; Fragaszy, Izar, Visalberghi, Ottoni \& Oliveira, 2004; Ottoni, Izar \& Resende, 2005). Apesar do uso de ferramentas ser documentado em várias espécies (incluindo aves, que além de usar ferramentas também as manufaturam - ver Hunt \& Gray, 2003), sua ocorrência associada a elementos como a aprendizagem por imitação e a formação de grupos, como acontece com os macacos-prego, pode ser propícia ao desenvolvimento de comportamento simbólico. Esse argumento torna favorável o uso do Cebus apella para estudar a aquisição de comportamento simbólico e pré-simbólico (Barros, Galvão \& McIlvane, 2003).

O que distingue os comportamentos simbólico e présimbólico é o tipo de relação de controle de estímulos. Discriminações simples e condicionais por identidade ou arbitrárias, por exemplo, podem ser considerados repertórios pré-simbólicos, envolvendo relações de controle de estímulo ensinadas diretamente. Desse treino direto podem emergir classes funcionais, um repertório simbólico no qual as relações de controle de estímulos não foram ensinadas diretamente, emergindo como produto das contingências de reforçamento (Sidman, 2000).

Barros, Galvão e McIlvane (2002), Galvão, Barros, Rocha, Mendonça e Goulart (2002), e Galvão e cols. (2005), ao estudar o comportamento de escolha em discriminações simples e condicionais com macacos-prego, relataram um procedimento geral que envolve a exposição gradual dos sujeitos a diferentes níveis de exigência de aprendizagem, inclusive para verificar e garantir a coerência entre as relações de controle de estímulos planejadas e aquelas aprendidas pelos sujeitos (ver também Barros \& cols., 2003; Barros, Galvão \& Rocha, 2005). Em todos esses estudos, os sujeitos foram submetidos inicialmente a tarefas mais simples (e.g., discriminações simples), para depois realizarem tarefas mais complexas (e.g., discriminações condicionais por identidade e arbitrárias).

Esses autores mostraram que os macacos-prego apresentavam indícios de identidade generalizada (IG) ${ }^{4} \mathrm{com}$

4 Apesar da aparente redundância do termo "identidade generalizada” (ver por exemplo Holth, 2003), utilizaremos aqui o termo “discriminação condicional por identidade” para repertórios diretamente treinados de escolha de acordo com o modelo, nos quais a comparação definida pelo experimentador como correta é idêntica ao modelo. A experiência de pesquisa tem mostrado que a maioria dos sujeitos, apesar de desempenhar esse tipo de tarefa com grande precisão após treino, não mantém os mesmos níveis de precisão quando estímulos novos são apresentados no mesmo tipo de tarefa. É possível que não apenas a identidade entre modelo e comparação correta esteja controlando o comportamento dos sujeitos durante o treino nesse caso ou que algum tipo de "neofobia" ocorra nas primeiras apresentações de estímulos completamente novos. O presente artigo inclusive é uma tentativa de elucidar parte das razões estímulos bidimensionais (figuras mostradas na tela de um computador), após treinos de reversões repetidas de discriminação simples (RRDS) ${ }^{5}$ e de discriminação condicional por identidade (DCI) também com figuras (Barros \& cols., 2002; Galvão \& cols. 2002; Galvão \& cols., 2005). Observou-se nesses estudos que os sujeitos apresentaram melhor desempenho nos testes de IG com as figuras que haviam sido utilizadas previamente em treinos de RRDS (Barros \& cols., 2002; Galvão \& cols., 2005).

Lima e cols. (2007) avaliaram a aplicação de um procedimento “curricular” para a aquisição dos repertórios de RRDS, DCI e IG com estímulos tridimensionais (objetos apresentados em bandeja). Dois macacos-prego (M09 e M13) foram submetidos inicialmente a treinos de RRDS com um conjunto de dois estímulos, seguidos por treino de DCI com outro conjunto de dois estímulos (para linha de base), e, por fim, a testes de IG. O primeiro teste foi feito com os estímulos usados no treino de RRDS e os outros com conjuntos novos. Ambos apresentaram indícios de aquisição de IG, mas apenas com estímulos utilizados previamente no treino de RRDS, replicando os resultados obtidos com estímulos bidimensionais (Barros \& cols., 2002; Galvão \& cols., 2005). Segundo Lima e cols., a aprendizagem das relações entre objetos foi aparentemente mais rápida que nos experimentos com figuras, apesar da dificuldade de se fazer comparação direta. Seus resultados sugeriram que o desempenho nas tarefas de RRDS, DCI e IG pode ser facilitado com o uso de objetos, com a realização prévia de tarefas com figuras e com treinos de RRDS com os estímulos de teste (a respeito do uso de objetos em tarefas de IG, ver também Kastak \& Schusterman, 1994).

Dois estudos com macacos-prego, um com figuras e outro com objetos, ambos usando procedimentos comparáveis de Discriminações Simples com Mudanças Sucessivas da função dos estímulos (DSMS - ver Nota 3), e de DCI, indicaram preliminarmente que com objetos a aprendizagem é mais rápida. Dahás e Souza (2005) treinaram dois macacos-prego ingênuos experimentalmente (M24 e M25) nessas duas tarefas, com quatro figuras, em pouco mais de 100 sessões. Souza e Fonseca (2006), utilizando outro macaco-prego (M18) que estava sendo submetido concomitantemente a um treino simultâneo de DSMS e de DCI com figuras (Rico, 2006), verificaram que o sujeito necessitou de menos de 50 sessões (22 para DSMS e 26 para DCI) para alcançar o critério de aprendizagem no mesmo procedimento utilizando objetos.

Esses resultados reforçaram a idéia de que o uso de objetos e o treino prévio (ou concomitante) nos procedimentos de DSMS e DCI com figuras podem facilitar a aquisição desses

pelas quais isso ocorre. Reservaremos o termo "identidade generalizada" para os casos em que esse repertório de escolha do estímulo de comparação idêntico ao modelo não se restringe aos exemplares diretamente treinados nesse tipo de contingência de reforçamento.

5 RRDS é um procedimento no qual dois estímulos ( $\mathrm{S}^{+}$e $\left.\mathrm{S}^{-}\right)$são apresentados ao sujeito e a escolha de somente um deles $\left(\mathrm{S}^{+}\right)$é reforçada. Quando o sujeito responde consistentemente ao $\mathrm{S}^{+}$, as funções de $\mathrm{S}^{+} \mathrm{e}$ $\mathrm{S}^{-}$são revertidas. Novamente, espera-se um bom desempenho para que outra reversão de funções ocorra. Quando são utilizados mais de dois estímulos, o termo mais adequado para descrever a mudança da função dos estímulos é Discriminações Simples com Mudanças Sucessivas nas funções dos estímulos (DSMS). 
repertórios. Considerando o desempenho de sujeito M18 nos treinos de DSMS e DCI com objetos e sua história de treino nessas tarefas também com figuras, ele se caracterizava como um excelente sujeito para avaliar de forma mais precisa os efeitos dessa história experimental sobre a aquisição de IG com objetos, usando uma metodologia do sujeito como seu próprio controle.

Com o objetivo de induzir o controle condicional por identidade, Rico (2006) introduziu adaptações no procedimento tradicional de DSMS (para uma descrição do procedimento tradicional, ver Nota 3), que consistiram na introdução de tentativas com apenas um estímulo (Tentativa Isolada), antecedendo tentativas com mais de um estímulo (Tentativa Simultânea). Na Tentativa Isolada era apresentado somente o estímulo idêntico ao que serviria como $\mathrm{S}^{+}$na Tentativa Simultânea. No decorrer do treino, era feita a introdução gradativa de novos S` nas Tentativas Simultâneas e o reforço na Tentativa Isolada era gradualmente removido, de forma que a Tentativa Isolada e a Tentativa Simultânea, tomadas em conjunto, emulavam as duas etapas de uma tarefa de DCI com atraso zero. Esperava-se que tal arranjo facilitasse o desenvolvimento de controle condicional por identidade. No estudo de Rico, com estímulos bidimensionais, a semelhança estrutural entre as sessões de DSMS adaptadas e DCI foi suficiente para que dois dos três animais que participaram do estudo apresentassem desempenho de DCI, mas não identidade generalizada.

No presente trabalho procurou-se estender os resultados prévios sobre a possibilidade de aquisição de IG com objetos por macacos-prego, avaliar a hipótese do efeito facilitador do treino prévio de DSMS com tentativas isoladas com os estímulos de teste de IG; e verificar se este efeito é função da exposição às contingências de alteração das funções dos estímulos ou simplesmente do contato com os estímulos, o que ameniza apenas o efeito de novidade da sua apresentação no teste de IG.

\section{Método}

\section{Sujeito}

Adam (M18), um macaco-prego (Cebus apella) macho, com 6 anos de idade e história prévia de treinos de DSMS com um conjunto de estímulos bidimensionais (figuras desenhadas em negro apresentadas dentro de uma janela de fundo branco de $2,23 \mathrm{~cm} 2$ - ver Rico, 2006) e de DCI com um conjunto tridimensional (o Conjunto A descrito no item Estímulos a seguir - ver Souza \& Fonseca, 2006). O sujeito habitava uma gaiola-viveiro, compartilhada com outros macacos da mesma espécie. Recebia uma refeição diária, basicamente constituída por frutas e ração com $28 \%$ de proteína. Além disso, recebia semanalmente suplementos aminoácidos, vitamínicos e minerais. $\mathrm{O}$ acesso à água era livre. Periodicamente era submetido a exames veterinários parasitológicos e infectológicos.

As condições de alojamento, manejo, alimentação e cuidados veterinários, bem como os procedimentos experimentais adotados no laboratório, foram aprovados pelo
Comitê de Ética em Pesquisa com Animais da Universidade Federal do Pará (CEPAE), mediante o documento CEPAEUFPA: PS001/2005, no que diz respeito à consonância com normas locais e internacionais para o tratamento e manipulação de animais de experimentação. A Escola Experimental de Primatas da UFPA está regularizada para criar animais silvestres (macacos) para pesquisa, fornecidos pelo próprio IBAMA e pelo Centro Nacional de Primatas, com Inscrição no IBAMA número 207419, Código Unidade 381201-4. O biotério é supervisionado pela Médica Veterinária Klena Sarges (CRMV-1210/PA-AP).

\section{Equipamento}

A câmara experimental media 0,60 x 0,60 x $0,60 \mathrm{~m}$, e ficava sobre uma mesa, em uma sala medindo 2,0 x 5,0 m, com duas lâmpadas fluorescentes de 40W. O experimentador permanecia ao lado da câmara durante as sessões. Um dos lados da câmara era um painel de alumínio que tinha uma abertura ao centro $(0,30 \times 0,23 \mathrm{~m})$ obstruída por quatro barras roliças de aço (de aproximadamente $4 \mathrm{~mm}$ de diâmetro), verticais e paralelas, separadas por intervalos de $5 \mathrm{~cm}$, que impediam o sujeito de sair da câmara. Acima da abertura ficavam três caixas de alumínio (5,0 x 7,0 x 2,0 cm), lado a lado e equidistantes, nas quais caíam, através de mangueiras, as pelotas liberadas por respectivos dispensadores acoplados à parte superior externa da câmara. Os outros lados e o teto eram de acrílico, encaixados na moldura de alumínio da câmara. No lado voltado para o centro da sala havia uma porta acrílica de correr, medindo $0,46 \times 0,30 \mathrm{~m}$, fechada externamente por um trinco, por onde o sujeito era introduzido e retirado da câmara. Nessa porta havia uma abertura retangular (5,0 x 25,0 cm) através da qual o sujeito podia pegar os objetos usados como estímulos. Uma chapa de alumínio retangular superposta à porta pelo seu lado externo era usada para bloquear o acesso pela abertura da porta quando necessário. Ao lado da porta havia uma abertura circular com $7,0 \mathrm{~cm}$ de diâmetro, na qual estava encaixada uma conexão do tipo joelho para tubos de PVC de $100 \mathrm{~mm}$, por onde o sujeito podia devolver os objetos, que eram apresentados sobre uma mesa de madeira sobre rodízios. A tampa da mesa movia-se sobre trilhos, para frente e para trás, permitindo a colocação dos objetos fora do alcance do sujeito e depois aproximá-la da abertura da porta da câmara. Na lateral da mesa havia um recipiente que, através de um plano inclinado de flandres, era conectado à extremidade do joelho de $\mathrm{PVC}$, possibilitando que o objeto devolvido pelo sujeito escorregasse para dentro do recipiente.

Sobre a mesa onde estava a câmara, ficava também um computador IBM (processador de $1.6 \mathrm{GHz}$ e Windows Xp Professional), no qual estava instalado o software VAICOM, utilizado para gerenciar o experimento. Havia ainda um monitor com tela sensível conectado ao computador. O software controlava a apresentação de figuras correspondentes aos objetos na tela em uma matriz de 3 x 3 (indicando ao experimentador como dispor os objetos sobre a mesa), controlava os dispensadores de pelotas e permitia registrar as respostas do sujeito (por meio de toques do experimentador nas figuras correspondentes aos objetos selecionados pelo sujeito). 

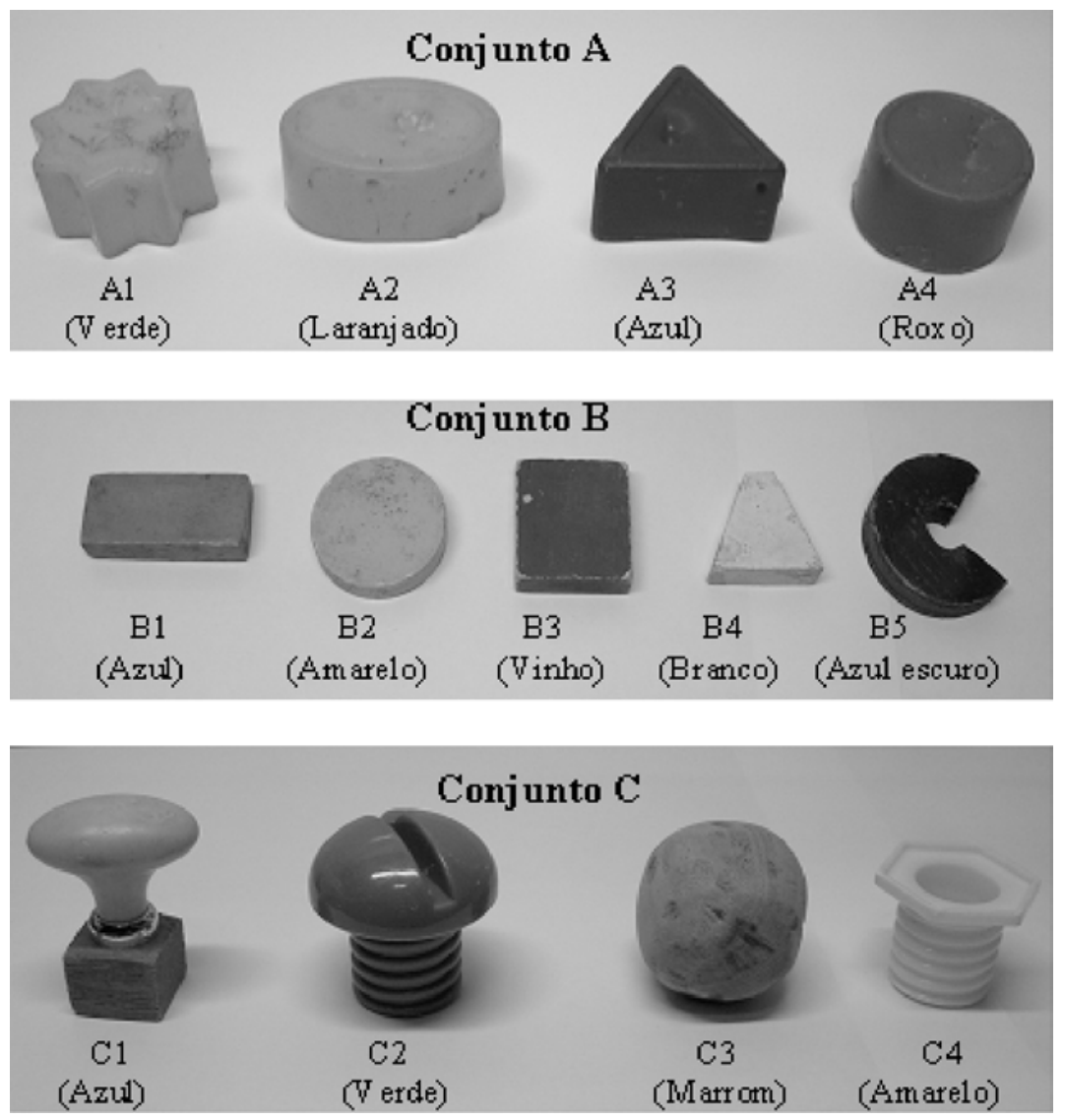

Figura 1. Conjuntos de estímulos usados no experimento. O estímulo B5 substituiu B4 na fase de preparação para o Teste 3 (ver resultados do Teste 3).

\section{Estímulos}

Foram utilizados três conjuntos (A, B e C) de quatro estímulos (Figura 1), os quais consistiam em objetos de formas e cores diferentes, de plástico ou de madeira e com até $5,0 \mathrm{~cm}$ em seu maior comprimento. O Conjunto A era composto pelos estímulos de linha de base do repertório de DCI, utilizados para a retomada do treino de DCI realizado por Souza e Fonseca (2006).

\section{Procedimento}

Foram conduzidos quatro testes de IG. Esses testes consistiam de uma sessão de DCI com 48 tentativas. A disposição dos objetos a cada tentativa era feita pelo experimentador de acordo com a configuração previamente programada no software VAICOM e mostrada na tela do computador. A tela do monitor era voltada para o experimentador e não visível para o sujeito. O experimentador colocava na mesa os estímulos indicados na tela, nas posições correspondentes. Uma tentativa tinha a seguinte sequência: um dos objetos (modelo) era colocado sobre a caixa móvel da mesa e então o sujeito podia pegá-lo pela abertura na porta da câmara e devolvê-lo pelo joelho de PVC. Os estímulos de comparação eram em seguida colocados sobre a mesa e, se o sujeito pegasse o objeto idêntico ao modelo $\left(\mathrm{S}^{+}\right)$, uma pelota era liberada pelo dispensador automático (mediante toque do experimentador na tela sobre a representação do estímulo correto, no momento que o sujeito devolvia o objeto pelo joelho de PVC) e tinha início um Intervalo Entre Tentativas (IET) de $5 \mathrm{~s}$. Caso o sujeito pegasse outro objeto ( $\mathrm{S}^{-}$), o experimentador tocava no estímulo correspondente na tela, a tentativa era encerrada e tinha início um IET de $5 \mathrm{~s}$. Nesse caso, uma nova tentativa só era iniciada após o sujeito ter devolvido o objeto S-

Teste 1. Consistiu em um teste padrão de IG, com estímulos nunca vistos pelo sujeito.

Preparação para o Teste 1. Foi realizada uma retomada do treino de DCI com os objetos do Conjunto A (A1, A2, A3 e A4). Em uma primeira etapa, as sessões foram constituídas por 40 tentativas divididas em dois blocos de 20 tentativas, com dois estímulos, A1 e A2. Cada estímulo (por exemplo, A1) aparecia como modelo em um bloco de 20 tentativas consecutivas, com duas comparações, A1 e A2. Em seguida o outro estímulo (por exemplo, A2) aparecia como modelo por 20 tentativas consecutivas. Na etapa seguinte, foram utilizados os estímulos A3 e A4, seguindo-se o mesmo procedimento. Em uma outra etapa, os quatro estímulos apareciam de forma randomizada em 40 tentativas (com cada estímulo aparecendo como modelo em 10 tentativas), ainda com duas comparações. Após isso, as tentativas passaram a ter três e depois quatro comparações, seguindo o mesmo 
Tabela 1. Etapas do treino de Discriminação Condicional de Identidade (DCI). Veja na Figura 1 a correspondência entre os códigos alfanuméricos e estímulos.

\begin{tabular}{clcl}
\hline $\begin{array}{c}\text { Número de } \\
\text { comparações }\end{array}$ & Modelos & $\begin{array}{c}\text { Número de } \\
\text { tentativas }\end{array}$ & Agrupamento de Tentativas \\
\hline 2 & A1, A2 & 40 & 2 blocos de 20 tentativas consecutivas \\
2 & A3, A4 & 40 & 2 blocos de 20 tentativas consecutivas \\
2 & A1, A2, A3, A4 & 40 & Tentativas randomizadas \\
3 & A1, A2, A3, A4 & 40 & Tentativas randomizadas \\
4 & A1, A2, A3, A4 & 40 & Tentativas randomizadas \\
\hline
\end{tabular}

procedimento, inclusive o critério para encerrar a etapa. O critério para encerramento da preparação para o teste foi o desempenho de duas sessões consecutivas com o mínimo de $90 \%$ de acerto na sessão geral e em cada bloco, ou seja, o sujeito precisava acertar nove das 10 tentativas de cada estímulo. Quando o sujeito alcançou o critério foi encerrada a fase de retomada da linha de base. Esse delineamento está resumido na Tabela 1.

Teste 1 propriamente dito. Depois que o sujeito atingiu o critério de aprendizagem na retomada do treino de DCI com os estímulos do Conjunto A, foi realizada a sessão de teste de IG. Essa sessão teve 48 tentativas, todas com quatro comparações, sendo 32 com estímulos do Conjunto A e 16 com estímulos novos do Conjunto B (B1, B2, B3, B4), e sendo quatro tentativas com cada estímulo como modelo, apresentadas de forma randômica. A resposta de escolha do sujeito na primeira tentativa de cada estímulo de teste não foi reforçada, o que possibilitou a emergência de identidade generalizada considerando principalmente as duas primeiras tentativas de cada relação e, adicionalmente, a manutenção do desempenho nas demais tentativas.

Teste 2. Foi realizado para averiguar se um treino prévio de indução de controle condicional via DSMS com os estímulos de teste facilitaria a ocorrência de IG.
Preparação para o Teste 2. O treino de indução de controle condicional via DSMS com os estímulos de teste (Conjunto B) foi feito de acordo com o procedimento desenvolvido por Rico (2006), descrito em linhas gerais na Introdução. No presente trabalho, o procedimento consistia na apresentação de uma Tentativa Isolada, na qual um estímulo era apresentado sozinho na mesa móvel em uma das nove posições possíveis indicadas pelo computador. Após o sujeito pegar o estímulo e devolvê-lo pelo tubo, essa resposta era reforçada com a apresentação de uma pelota de comida (o experimentador tocava no estímulo apresentado na tela e o dispensador era acionado). Em seguida, o mesmo estímulo, com função de $\mathrm{S}^{+}$, era apresentado ao sujeito juntamente com outro estímulo como S- (Tentativa Simultânea). Se o sujeito escolhesse o correto e o devolvesse, recebia mais uma pelota de comida após toque do experimentador na tela. Se pegasse o outro, a tentativa era encerrada sem a apresentação de pelota de comida. Uma nova Tentativa Isolada era apresentada em seguida. A sessão era constituída por até 40 tentativas. Se o sujeito atingisse o critério de oito respostas corretas consecutivas (cc) nas Tentativas Simultâneas, era encerrada uma etapa, conforme descrito a seguir.

A modelagem de DSMS com o Conjunto B foi constituída por 13 etapas (ver Tabela 2). Da $1^{\mathrm{a}}$ à $9^{\mathrm{a}}$ etapa, novos estímulos foram adicionados às sessões, inicialmente com função de

Tabela 2. Estímulos usados nas etapas do Treino de Discriminações Simples com Mudanças Sucessivas (DSMS). Veja na Figura 1 a correspondência entre os códigos alfanuméricos e estímulos.

\begin{tabular}{llllll}
\hline \multirow{2}{*}{ Etapa } & \multicolumn{5}{c}{ Tentativa } \\
\cline { 2 - 6 } & & \multicolumn{3}{c}{ Simultânea } \\
\cline { 2 - 5 } & & Isolada & $\mathbf{S}^{+}$ & $\mathbf{S}^{-}$ & \\
\hline 1 & B1 & B1 & B2 & - & - \\
2 & B2 & B2 & B1 & - & - \\
3 & B2 & B2 & B1 & B3 & - \\
4 & B3 & B3 & B1 & B2 & - \\
5 & B1 & B1 & B2 & B3 & - \\
6 & B1 & B1 & B2 & B3 & B4 \\
7 & B4 & B4 & B2 & B3 & B1 \\
8 & B3 & B3 & B2 & B1 & B4 \\
9 & B2 & B2 & B1 & B3 & B4 \\
10 & B2 & B2 & B1 & B3 & B4 \\
11 & B3 & B3 & B1 & B2 & B4 \\
12 & B4 & B4 & B1 & B2 & B3 \\
13 & B1 & B1 & B2 & B3 & B4 \\
\hline
\end{tabular}


S-, e a função dos estímulos mudava cada vez que o sujeito alcançava o critério de 8cc. Isso foi feito até todos os quatro estímulos passarem por uma etapa com o $\mathrm{S}^{+}$, acompanhados de três $\mathrm{S}^{-}$nas tentativas simultâneas.

As etapas 10 a 13 consistiram na diminuição gradual da probabilidade de reforçamento para as respostas das tentativas isoladas. Essas quatro etapas com redução da probabilidade de reforço tiveram a função de fazer aproximação do procedimento de DSMS com o de DCI para a realização da fase de teste.

Passada essa fase, foi feita a retomada da linha de base de DCI com os estímulos do Conjunto A (A1 A2, A3 e A4), que havia sido estabelecida anteriormente na preparação para o Teste 1 . A retomada foi feita em sessões de 40 tentativas com quatro comparações, com critério de duas sessões consecutivas com $90 \%$ de acerto em cada estímulo. Até o sujeito alcançar o critério, foram feitas sessões intercaladas de DSMS com o Conjunto B.

Teste 2 propriamente dito. O teste foi feito em uma sessão de 48 tentativas com quatro comparações, sendo 32 de linha de base (Conjunto A) e 16 de teste (Conjunto B), e sendo quatro com cada estímulo modelo. A resposta de escolha do sujeito na primeira tentativa de cada estímulo de teste não foi reforçada e o critério de aprendizagem foi o mesmo do Teste 1 .

Teste 3. Esse teste foi feito para verificar se apenas o contato direto prévio do sujeito com os estímulos de teste, sem nenhuma contingência de reforçamento programada, poderia facilitar a tarefa proposta.

Preparação para o Teste 3. Foram utilizados quatro novos estímulos: C1, C2, C3 e C4. Primeiro, o sujeito passou por uma exposição aos estímulos com livre manipulação dos mesmos. Isto é, um dos novos estímulos foi entregue ao sujeito dentro da câmara experimental, permitindo a sua livre manipulação pelo sujeito por um período de aproximadamente 5 minutos. Após esse período, o estímulo era recolhido e um novo estímulo era entregue ao sujeito para livre manipulação por outros 5 minutos, e assim sucessivamente, até que os quatros estímulos do Conjunto $\mathrm{C}$ tivessem sido manipulados pelo sujeito.

A etapa seguinte foi a retomada da linha de base em DCI com os estímulos dos conjuntos A e B (40 tentativas, 20 para cada conjunto, cinco de cada estímulo) com quatro comparações e com o mesmo critério de duas sessões com 90\% de acerto em cada estímulo.

Teste 3 propriamente dito. O teste foi feito em uma sessão com 48 tentativas das quais 16 eram de linha de base do Conjunto A, 16 de linha de base do Conjunto B e 16 tentativas de teste com os estímulos manipulados pelo sujeito (Conjunto C). Foram estabelecidas as mesmas condições de reforçamento e mesmo critério de acerto dos testes 1 e 2 .

Teste 4. Procurou avaliar a aquisição de IG com os estímulos usados no Teste 3 após realizar treino de indução de controle condicional (DSMS com tentativa isolada) com esses estímulos.

Preparação para o Teste 4. Idêntica à do Teste 2, mas utilizando os estímulos do Conjunto C no treino de DSMS e os dos conjuntos A e B na retomada do treino de DCI.

Teste 4 propriamente dito. Idêntico ao Teste 3.

\section{Resultados e Discussão}

O desempenho do sujeito alcançou critério de acerto nos testes 2 e 4. Esses resultados reforçam aqueles que apontam que macacos-prego podem adquirir IG com objetos (Lima \& cols., 2007).

O sujeito necessitou de 98 sessões de retomada de linha de base em preparação para o Teste 1 . Na sessão de teste, das 16 tentativas com estímulos novos, o sujeito acertou duas. Considerando o desempenho do sujeito nas duas primeiras tentativas de cada relação, houve dois acertos em oito tentativas. Houve também uma queda no desempenho nas tentativas de linha de base. Das 32 tentativas, o sujeito acertou 26. A Tabela 3 mostra o desempenho no Teste 1 . A escolha de apresentação dos dados tentativa a tentativa (sem a convencional apresentação de figuras com dados quantitativos totais) está relacionada ao critério de avaliação do desempenho nos testes, pioneiramente apresentado por Schusterman e Kastak (1993). De acordo com o critério adotado naquele estudo, é importante averiguar se houve escolha correta na primeira tentativa (e no presente caso na segunda tentativa também) de cada relação testada. A manutenção do desempenho nas demais tentativas do teste apenas confirma a emergência.

Esses dados apontam que testes de IG com estímulos completamente novos para o sujeito podem não ser adequados para avaliar a aquisição desse repertório em macacos-prego, mesmo em um sujeito com uma história prévia de treinos de DSMS e DCI com figuras e objetos. Barros e cols. (2002), Galvão e cols. (2005), e Lima e cols. (2007), entretanto, relataram que a utilização dos estímulos de teste de IG em

Tabela 3. Sequência de acertos (C) e erros (X) em cada discriminação do Teste 1 de Identidade Generalizada. Ao lado de cada indicação de erro nas tentativas de teste, o estímulo selecionado é apresentado entre parênteses. Veja na Figura 1 a correspondência entre os códigos alfanuméricos e estímulos.

\begin{tabular}{|c|c|c|c|c|}
\hline \multirow{2}{*}{$\begin{array}{l}\text { Tentativa } \\
\text { de Teste }\end{array}$} & \multicolumn{4}{|c|}{ Estímulo Comparação Correto } \\
\hline & B1 & B2 & B3 & B4 \\
\hline 1 & $\mathrm{X}(\mathrm{B} 2)$ & $\mathrm{C}$ & X (B2) & C \\
\hline 2 & $\mathrm{X}(\mathrm{B} 3)$ & $\mathrm{X}(\mathrm{B} 4)$ & $\mathrm{X}(\mathrm{B} 4)$ & $\mathrm{X}(\mathrm{B} 3)$ \\
\hline 3 & $\mathrm{X}(\mathrm{B} 2)$ & $\mathrm{X}(\mathrm{B} 4)$ & $\mathrm{X}(\mathrm{B} 1)$ & $\mathrm{X}(\mathrm{B} 1)$ \\
\hline 4 & $\mathrm{X}(\mathrm{B} 4)$ & X (B3) & $\mathrm{X}(\mathrm{B} 4)$ & $\mathrm{X}(\mathrm{B} 2)$ \\
\hline $\begin{array}{c}\text { Tentativa } \\
\text { de LB }\end{array}$ & A1 & A2 & A3 & A4 \\
\hline 1 & $\mathrm{C}$ & $\mathrm{C}$ & $\mathrm{C}$ & $\mathrm{C}$ \\
\hline 2 & $\mathrm{x}$ & $\mathrm{C}$ & $\mathrm{C}$ & $\mathrm{X}$ \\
\hline 3 & $\mathrm{C}$ & C & $\mathrm{C}$ & $\mathrm{C}$ \\
\hline 4 & $\mathrm{C}$ & $\mathrm{x}$ & C & $\mathrm{C}$ \\
\hline 5 & $\mathrm{x}$ & C & $\mathrm{C}$ & $\mathrm{C}$ \\
\hline 6 & $\mathrm{C}$ & $\mathrm{C}$ & $\mathrm{C}$ & $\mathrm{C}$ \\
\hline 7 & $\mathrm{C}$ & $\mathrm{C}$ & $\mathrm{C}$ & $\mathrm{C}$ \\
\hline 8 & C & $\mathrm{X}$ & $X$ & C \\
\hline
\end{tabular}


Tabela 4. Sequência de acertos (C) e erros (X) em cada discriminação do Teste 2 de Identidade Generalizada com o Conjunto B de objetos, feito depois de treino de DSMS com o Conjunto B. Ao lado de cada indicação de erro nas tentativas de teste, o estímulo selecionado é apresentado entre parênteses. Veja na Figura 1 a correspondência entre os códigos alfanuméricos e estímulos.

\begin{tabular}{|c|c|c|c|c|}
\hline \multirow{2}{*}{$\begin{array}{l}\text { Tentativa } \\
\text { de Teste }\end{array}$} & \multicolumn{4}{|c|}{ Estímulo Comparação Correto } \\
\hline & B1 & B2 & B3 & B4 \\
\hline 1 & $\mathrm{C}$ & $\mathrm{C}$ & $\mathrm{C}$ & $\mathrm{C}$ \\
\hline 2 & $\mathrm{C}$ & $\mathrm{C}$ & $\mathrm{C}$ & $\mathrm{C}$ \\
\hline 3 & $\mathrm{C}$ & $\mathrm{C}$ & $\mathrm{X}(\mathrm{B} 1)$ & $\mathrm{C}$ \\
\hline 4 & $\mathrm{C}$ & $\mathrm{C}$ & $\mathrm{X}(\mathrm{B} 1)$ & $\mathrm{X}(\mathrm{B} 1)$ \\
\hline $\begin{array}{c}\text { Tentativa } \\
\text { de LB }\end{array}$ & A1 & A2 & A3 & A4 \\
\hline 1 & $\mathrm{C}$ & $\mathrm{C}$ & $\mathrm{C}$ & $\mathrm{C}$ \\
\hline 2 & $\mathrm{C}$ & $\mathrm{C}$ & $\mathrm{X}$ & $\mathrm{C}$ \\
\hline 3 & $\mathrm{C}$ & $\mathrm{C}$ & $\mathrm{C}$ & $\mathrm{C}$ \\
\hline 4 & $\mathrm{C}$ & $\mathrm{C}$ & $\mathrm{C}$ & $\mathrm{C}$ \\
\hline 5 & $\mathrm{X}$ & $\mathrm{C}$ & $\mathrm{C}$ & $\mathrm{C}$ \\
\hline 6 & $\mathrm{C}$ & $\mathrm{C}$ & $\mathrm{C}$ & $\mathrm{C}$ \\
\hline 7 & $\mathrm{C}$ & $\mathrm{X}$ & $X$ & $\mathrm{C}$ \\
\hline 8 & $\mathrm{C}$ & X & $\mathrm{C}$ & $\mathrm{C}$ \\
\hline
\end{tabular}

treinos prévios de DSMS poderia facilitar a aquisição da IG, de forma que, no Teste 2, essa possibilidade foi avaliada.

Após o treino de DSMS com tentativas isoladas e com dois estímulos alternados (uma sessão para cada etapa), e de retomada de DCI (10 sessões), o sujeito realizou com sucesso o Teste 2. Foram registrados 13 acertos nas 16 tentativas de teste. Houve $100 \%$ de acertos nas duas primeiras tentativas de cada relação, como mostra a Tabela 4 . Nas tentativas de linha de base, ele errou apenas cinco das 32.

Com os mesmos estímulos do Teste 1 , no qual o sujeito não alcançou o critério de acerto, no Teste 2, após treino de DSMS, ele alcançou esse critério. Esse resultado corrobora dados de Barros e cols. (2002), Galvão e cols. (2005), e Lima e cols. (2007), que indicaram o efeito facilitador do treino prévio de DSMS com os estímulos a serem usados nos testes de IG com macacos-prego. Contudo, ainda não estava claro, até esse ponto, se esse efeito era função da exposição às contingências de alteração das funções dos estímulos ou simplesmente do contato com os estímulos, o que amenizaria o efeito de novidade da apresentação dos mesmos no teste de IG. O Teste 3 avaliou essa questão.

O treino prévio ao Teste 3 envolveu a livre manipulação dos estímulos do Conjunto C, o que durou quatro sessões. A retomada de linha de base levou cinco sessões, sendo que, a partir da terceira sessão, o estímulo B4 foi substituído por B5 em função do baixo desempenho nas tentativas com ambos. Na sessão de teste, o sujeito não alcançou o critério, uma vez que foram registrados apenas quatro acertos nas oito tentativas críticas (duas primeiras de cada relação). Houve
11 acertos nas 16 tentativas de teste. O desempenho na linha de base foi preciso em 13 das 16 tentativas do Conjunto A e 11 das 16 do Conjunto B (Tabela 5).

Esse resultado indica que a simples exposição prévia aos estímulos de teste não foi suficiente para a ocorrência de IG, ainda que tenha favorecido o seu desenvolvimento, se forem considerados os 11 acertos nas 16 tentativas de teste, com $100 \%$ de acerto para um dos estímulos. Esses dados sugerem que o efeito de novidade dos estímulos no teste não é o único empecilho à pronta demonstração do repertório de IG. Parece ser importante que os estímulos de teste tenham estado presentes em situações prévias de exposição a contingências de reforçamento (em situações de DSMS ou de discriminação simples - ver Teste 4 e Considerações Finais a seguir).

Para confirmar o efeito do treino prévio de DSMS sobre a aquisição de IG, foi realizado o Teste 4, no qual foi feito treino de DSMS com o Conjunto $\mathrm{C}$ e o sujeito realizou cada etapa em uma sessão. Na retomada de DCI com os conjuntos A e B, o sujeito levou 13 sessões para alcançar o critério. Na sessão de teste, foram registrados 14 acertos nas 16 tentativas com os estímulos de teste. Considerando o desempenho do sujeito nas duas primeiras tentativas de cada relação, houve sete acertos em oito tentativas (o desempenho só não foi preciso na segunda tentativa da relação C1C1). Nas tentativas com os estímulos de linha de base, o sujeito acertou 12 tentativas do Conjunto A e 14 do Conjunto B (ver Tabela 6).

O desempenho do sujeito no Teste 4 sugere emergência de identidade generalizada com sete acertos em oito

Tabela 5. Sequência de acertos (C) e erros (X) em cada discriminação do Teste 3 de Identidade Generalizada, feito após quatro sessões de manipulação dos objetos usados no teste. Ao lado de cada indicação de erro nas tentativas de teste, o estímulo selecionado é apresentado entre parênteses. Veja na Figura 1 a correspondência entre os códigos alfanuméricos e estímulos.

\begin{tabular}{ccccc}
\hline \multirow{2}{*}{$\begin{array}{c}\text { Tentativa } \\
\text { de Teste }\end{array}$} & \multicolumn{4}{c}{ Estímulo Comparação Correto } \\
\cline { 2 - 5 } & C1 & C2 & C3 & C4 \\
\hline 1 & X (C3) & C & C & X (C2) \\
2 & X (C2) & C & X (C2) & C \\
3 & C & C & C & C \\
4 & X (C4) & C & C & C \\
\hline Tentativa & A1 & A2 & A3 & A4 \\
\hline de LB A & X & C & C & C \\
\hline 1 & X & X & C & X \\
2 & C & C & C & X \\
3 & C & C & C & C \\
4 & B1 & B2 & B3 & B5 \\
\hline Tentativa \\
de LB B
\end{tabular}


Tabela 6. Sequência de acertos (C) e erros (X) em cada discriminação do Teste 4 de Identidade Generalizada. Ao lado de cada indicação de erro nas tentativas de teste, o estímulo selecionado é apresentado entre parênteses. Veja na Figura 1 a correspondência entre os códigos alfanuméricos e estímulos.

\begin{tabular}{ccccc}
\hline \multirow{2}{*}{$\begin{array}{c}\text { Tentativa } \\
\text { de Teste }\end{array}$} & \multicolumn{4}{c}{ Estímulo Comparação Correto } \\
\cline { 2 - 5 } & C1 & C2 & C3 & C4 \\
\hline 1 & C & C & C & C \\
2 & X (C4) & C & C & C \\
3 & C & C & C & C \\
4 & C & X (C4) & C & C \\
\hline Tentativa & A1 & A2 & A3 & A4 \\
\hline 1 & C & X & C & C \\
2 & C & C & C & C \\
3 & C & C & C & X \\
4 & C & C & C & C \\
\hline $\begin{array}{c}\text { Tentativa } \\
\text { de LB B }\end{array}$ & B1 & B2 & B3 & B5 \\
\hline 1 & C & C & C & X \\
2 & X & C & C & C \\
3 & C & C & C & X \\
4 & X & C & C & C \\
\hline
\end{tabular}

nas tentativas críticas e com manutenção de desempenho nas demais tentativas (14 acertos em 16 tentativas). Esse resultado reforça a idéia de que uma exposição prévia às contingências de reforçamento em treinos de RRDS/DSMS favorece a aquisição de IG.

\section{Considerações Finais}

O sujeito apresentou bom desempenho nos treinos de DCI e DSMS com objetos, com tentativas isoladas e simultâneas alternadas, atingindo critério de aprendizagem rapidamente. A aquisição desses repertórios ocorreu em um número menor de sessões que com os macacos-prego ingênuos submetidos a essas mesmas tarefas com estímulos bidimensionais (Dahás \& Souza, 2005).

Os testes nos quais o sujeito teve melhor desempenho foram precedidos de treino de indução de controle condicional com os estímulos de teste. Embora seja esperado que a apresentação do $\mathrm{S}^{+}$em tentativa isolada imediatamente antes da tentativa de DSMS favoreça o desenvolvimento de controle relacional, é possível que cada nova mudança de função seja resolvida estritamente como uma tarefa de DSMS, sem qualquer participação do "modelo” da tentativa isolada. Nesse caso, o efeito aparentemente facilitador do contato prévio com os estímulos de teste em DSMS seria da mesma natureza do observado em estudos anteriores (Barros \& cols., 2002; Galvão \& cols., 2005; Lima \& cols., 2007). Permanece por ser avaliado se o efeito da exposição prévia às contingências de reforçamento depende da simples exposição a situações de discriminação simples (DS), com ou sem tentativas isoladas, ou das sucessivas mudanças das funções dos estímulos que ocorre no treino de DSMS. Pode-se considerar, a princípio, que as alterações das funções dos estímulos dos treinos de DSMS podem preparar melhor os sujeitos para a situação de pareamento ao modelo que caracteriza os testes de IG, uma vez que esse efeito foi documentado por Barros e cols. (2002) e Galvão e cols. (2005), sem o uso de tentativa isolada.

Recentemente, Beran e cols. (2008) demonstraram que, no treino de reversão de discriminação simples com duas escolhas com macacos-prego, pode prevalecer a rejeição do estímulo com função negativa ( $\left.\mathrm{S}^{-}\right)$no treino anterior, corroborando os dados de Goulart, Mendonça, Barros, Galvão e McIlvane (2005) sobre o estabelecimento de controle por seleção (escolha do $\mathrm{S}^{+}$) ou rejeição (rejeição do $\mathrm{S}^{-}$) em treinos de discriminação simples com macacos-prego. No presente estudo, o uso de quatro estímulos de escolha (tanto como comparação no procedimento de pareamento ao modelo, quanto nas tentativas simultâneas nos treinos de DSMS) pode ter minorado as chances de controle pelo estímulo $\mathrm{S}^{-}$. Se isso de fato ocorreu, a eficácia do treino de DSMS pode ter sido aumentada no presente experimento, na medida em que o estímulo controlador era de fato aquele em relação ao qual o experimentador planejava prover experiência ao sujeito.

Há também a possibilidade de que a exposição sucessiva a procedimentos de discriminação simples (DS) e de DCI seja, por si mesma, uma variável que possibilite a aquisição de IG. Isto é, que o sujeito aprenda a responder a estímulos novos sem a necessidade de ser sempre submetido a outros treinos com os estímulos de teste. Esse processo poderia ser caracterizado como learning set (Harlow, 1949).

Desta forma, novos estudos que procurem avaliar a aquisição de IG em macacos-prego poderiam investigar aspectos tais como: (1) qual o efeito de treinos prévios em DS com os estímulos a serem usados em testes de IG; (2) se treinos sucessivos de DCI e DSMS com tentativa isolada com estímulos que não serão usados nos testes podem produzir a IG.

\section{Referências}

Barros, R. S., Galvão, O. F., \& McIlvane, W. J. (2002). Generalized identity matching-to-sample in Cebus apella. The Psychological Record, 52, 441-460.

Barros, R. S., Galvão, O. F., \& McIlvane, W. J. (2003). The search for relational learning capacity in Cebus apella: A programmed “educational” approach. Em S. Soraci Jr. \& K. Murata-Soraci (Eds.), Visual information processing (pp. 223-245). Westport: Praeger Publishers.

Barros, R. S., Galvão, O. F., \& Rocha, A. C. (2005). O pesquisador na escola experimental de primatas: de experimentador a programador de contingências. Interação em Psicologia, 9, 201 -214 .

Beran, M. J., Klein, E. D., Evans, T. A., Chan, B., Flemming, T. M., Harris, E. H., Washburn, D. A., \& Rumbaugh, D. M. (2008). Discrimination reversal learning in capuchin monkeys (Cebus apella). The Psychological Record, 58, 3-14. 
Dahás, L. J. S., \& Souza, C. B. A. (2005). Aquisição de repertório simbólico em macaco-prego (Cebus apella). Relatório de Pesquisa. PROPESP/UFPA.

Evans, T. A., \& Westergaard, G. C. (2004). Discrimination of functionally appropriate and inappropriate throwing tools by captive tufted capuchins (Cebus apella). Animal Cognition, 7, 255-262.

Fragaszy, D. M., Izar, P., Visalberghi, E., Ottoni, E. B., \& Oliveira, M. G. (2004). Wild capuchin monkeys (Cebus libidinosus) use anvils and stone pounding tools. American Journal of Primatology, 64, 359-366.

Galvão, O. F., Barros, R. S., Rocha, A. C., Mendonça, M., \& Goulart, P. R. K. (2002). Escola experimental de primatas. Estudos de Psicologia, 7, 361-370.

Galvão, O. F., Barros, R. S., Santos, J. R., Brino, A. L. F., Brandão, S., Lavratti, C. M., Dube, W. V., \& McIlvane, W. J. (2005). Extent and limits of the matching concept in Cebus apella: A matter of experimental control? The Psychological Record, 55, 219-232.

Goulart, P. R. K., Mendonça, M. B., Barros, R. S., Galvão, O. F., \& McIlvane, W. J. (2005). A note on select- and reject-controlling relations in the simple discrimination of capuchin monkeys (Cebus apella). Behavioural Processes, 69, 295-302.

Harlow, H. F. (1949). The formation of learning sets. Psychological Review, 56, 51-65.

Hauser, M. D., Chomsky, N., \& Fitch, W. T. (2002). The faculty of language: What is it, who has it, and how did it evolve? Science, 298, 1569-1579.

Herman, L. M., Gory, J. D., Hovancik, J. R., \& Bradshaw, G. L. (1989). Generalization of visual matching by a bottlenosed dolphin (Tursiops truncatus): Evidence for invariance of cognitive performance with visual and auditory materials. Animal Behavior Processes, 15, 124-136.

Hixson, M. D. (1998). Ape language research: A review and behavioral perspective. The Analysis of Verbal Behavior, 15, 17-39.

Holth, P. (2003). Generalized imitation and generalized matching to sample. The Behavior Analyst, 26, 155- 158.

Hunt, G. R., \& Gray, R. D. (2003). Diversification and cumulative evolution in New Caledonian crow tool manufacture. Proceedings of the Royal Society B: Biological Sciences, 270, 867-874.

Kastak, D., \& Schusterman, R. J. (1994). Transfer of visual identity matching-to-sample in two California sea lions (Zalophus californianus). Animal Learning \& Behavior, 22, 427-435.

Lima, M. E. A. C., Barros, R. S., Dahás, L. J. S., Cruz, A. P. C., Bezerra, D. S., \& Galvão, O. F. (2007). Discriminação simples e pareamento ao modelo por identidade com estímulos tridimensionais em macacos-prego (Cebus apella). Acta Comportamentalia, 15, 5-20.
McIlvane, W. J., \& Cataldo, M. (1996). On the clinical relevance of animal models for the study of mental retardation. Mental Retardation Developmental Disabilities Research Review, 2, 188-196.

Ottoni, E. B., Izar, P., \& Resende, B. D. (2005). Watching the best nutcrackers: What capuchin monkeys (Cebus apella) know about others' tool-using skills. Animal Cognition, 24, 215-219.

Rico, V. (2006). Persistência comportamental e topografia de controle de estímulos coerente em treino de discriminação simples e escolha condicional por identidade ao modelo com quatro escolhas em macacos-prego. Dissertação de Mestrado, Universidade Federal do Pará, Belém.

Savage-Rumbaugh, E. (1984). Verbal behavior at a procedural level in the chimpanzee. Journal of the Experimental Analysis of Behavior, 41, 223-250.

Savage-Rumbaugh, E., \& Lewin, R. (1994). Kanzi: The ape at the brink of the human mind. New York: John Wiley \& Sons.

Savage-Rumbaugh, S., McDonald, R., Sevcik, R. A., Hopkins, W. D., \& Rubert, E. (1986). Spontaneous symbol acquisition and communicative use by pygmy chimpanzees (Pan paniscus). Journal of Experimental Psychology: General, 115, 211-235.

Savage-Rumbaugh, E., Murphy, J., Sevcik, R., Brakke, K., Williams, S., \& Rumbaugh, D. (1993). Language comprehension in ape and child. Monographs of the Society for Research in Child Development, 58, 1-256.

Schusterman, R. J., \& Kastak, D. (1993). A california sea lion (Zalophus californianus) is capable of forming equivalence relations. The Psychological Record, 43, 823-844.

Sidman, M. (2000). Equivalence relations and the reinforcement contingency. Journal of the Experimental Analysis of Behavior, 74, 127-146.

Souza, C. B. A., \& Fonseca, A. R. (2006). Aquisição de repertório simbólico em macaco-prego (Cebus apella). Relatório de Pesquisa. PROPESP/UFPA.

Tomasello, M., \& Call, J. (1997). Primate cognition. New York: Oxford University Press. 